\title{
Análisis de la demanda residencial de los servicios básicos en España usando un modelo QUAIDS censurado*
}

\author{
Analysis of the residential demand of basic services in Spain using a censored \\ QUAIDS model
}

\author{
Pablo GálveZ** \\ Petr MARIEL *** \\ DAVID HoYOS****
}

\begin{abstract}
Resumen
El presente artículo analiza la demanda residencial española en los bienes que forman parte de los servicios básicos del hogar (electricidad, gas natural y agua potable) en 2012. El análisis se realiza utilizando un modelo QUAIDS adaptado para tratar la censura en el consumo de gas natural según el enfoque de Tauchmann (2010), uno de los últimos avances en esta materia. Los resultados muestran que la demanda de electricidad y agua potable es menos sensible a las variaciones de sus precios e ingreso residencial que la demanda de gas natural.

Palabras clave: Electricidad; gas natural; agua potable; modelo QUAIDS censurado.

Clasificación JEL: $C 3, Q 2, Q 3, Q 4$.
\end{abstract}

\begin{abstract}
This paper analyses the Spanish residential demand of a bundle of goods conforming the basic services of the households (i.e. electricity, natural gas and drinking water) in 2012. The analysis is made using a QUAIDS model modified in order to consider the censoring in the consumption of natural gas following the approach of Tauchmann (2010), one of the latest advances in this field. The

* Los autores agradecen el apoyo financiero tanto del Gobierno Vasco como de la UPV/ EHU mediante los proyectos IT-642-13, UFI11/03, US12/09 y US15/11. Además, los autores agradecen al editor y a los revisores anónimos sus comentarios acerca del trabajo. Sus sugerencias nos han permitido mejorar sustancialmente la calidad del mismo.

** Escuela Universitaria de Ingeniería Industrial, Informática y de Sistemas, Universidad de Tarapacá (UTA), Chile. Email: pgalvez@uta.cl.

*** Departamento de Economía Aplicada III, Facultad de Ciencias Económicas y Empresariales, Universidad del País Vasco (UPV/EHU), España. Email: petr.mariel@ehu.eus

***** Departamento de Economía Aplicada III, Facultad de Ciencias Económicas y Empresariales, Universidad del País Vasco (UPV/EHU), España. Email: david.hoyos@ehu.eus
\end{abstract}


results show that the demand for electricity and drinking water is less sensitive to the variations in prices and household's income than that of natural gas.

Key words: Electricity; natural gas; drinking water; censored QUAIDS model.

JEL Classification: $C 3, Q 2, Q 3, Q 4$.

\section{INTRODUCCIÓN}

El análisis de la demanda residencial de los bienes que forman los servicios básicos del hogar (electricidad, gas y agua potable) es importante debido a su relación con el bienestar de una familia y su vulnerabilidad. En España, una familia es vulnerable si no puede costear sus servicios básicos y se acoge al bono social en el consumo eléctrico y de gas natural (BOCG, 2009; BOE, 2012).

Lamentablemente, la situación de vulnerabilidad de las familias españolas se ha incrementado desde el inicio de la crisis económica en 2008. Algunas cifras indican que en 2012 el ingreso medio de las familias cae 10,9\% respecto del inicio de la crisis. El $20 \%$ de los hogares se encuentra en riesgo de pobreza y el 8,4\% reconoce tener retrasos en los pagos relacionados con la vivienda principal, entre los que destacan el gasto en la energía y agua potable (INE, 2013).

Por otro lado, los precios de la electricidad y el gas natural, que son los principales combustibles residenciales españoles (IDAE, 2011), han experimentado un notable crecimiento. En 2012 los hogares españoles enfrentan un incremento del $10,6 \%$ y $23,4 \%$ en el precio de la electricidad y gas natural, respectivamente. Esto significa que los hogares españoles pagan $32 \%$ más por electricidad y 15,2\% más por gas natural que el promedio de los países de la Unión Europea (UE) (Eurostat, 2014). En relación con el agua potable, el índice de precios al consumidor de la distribución de agua aumenta 5,1\% respecto del año anterior y $16,1 \%$ respecto del inicio de la crisis económica INE (2014).

En relación con la literatura sobre el consumo de los bienes que forman los servicios básicos, existe una gran cantidad de trabajos que estudian en consumo residencial de energía, pero, a nuestro saber, no incorporan el consumo de agua potable. Además, los artículos que analizan la situación española sobre la demanda residencial de energía y de agua potable siguen siendo escasos.

Por tal motivo, el objetivo de este artículo consiste en analizar la demanda residencial española de electricidad, gas natural y agua potable de forma conjunta. Para ello se utilizan los datos que proporciona la Encuesta de Presupuestos Familiares (EPF) de 2012 junto con un sistema de ecuaciones llamado QUAIDS (del inglés, Quadratic Almost Ideal Demand System). Este artículo pone especial énfasis en la obtención de las elasticidades de precio e ingreso de la demanda residencial de electricidad, gas natural y agua potable. Estos resultados pueden contribuir al diseño de políticas que promuevan un uso más eficiente de la energía y agua potable residencial.

Además de la contribución a la literatura acerca de la demanda residencial de los servicios básicos, este artículo destaca por utilizar uno de los últimos avances en el tratamiento de la censura en un sistema de ecuaciones. Es importante mencionar que dicha censura surge porque no todos los hogares españoles consumen gas natural. En particular, se aplica el enfoque propuesto por Tauchmann (2010) 
y, hasta donde sabemos, este trabajo es uno de los primeros que incorpora este tratamiento en un modelo QUAIDS.

Incluyendo la Introducción, el presente artículo está formado por siete secciones. La Sección 2 contiene una breve revisión de literatura respecto de la demanda residencial de electricidad, gas natural y agua potable. La Sección 3 resume los principales enfoques para tratar la censura en un modelo de sistema de ecuaciones. La Sección 4 muestra el enfoque propuesto por Tauchmann (2010) para el tratamiento de la censura e indica una importante contribución de este artículo: cómo se adapta dicho enfoque cuando existe solo un bien censurado en un modelo QUAIDS. La Sección 5 presenta los datos y variables del estudio. La Sección 6 indica los resultados obtenidos y las estimaciones de las elasticidades precio, gasto e ingreso de la demanda residencial en electricidad, gas natural y agua potable. Finalmente, la Sección 7 presenta las principales conclusiones.

\section{REVISIÓN DE LITERATURA}

La literatura dedicada al análisis de la demanda residencial de electricidad y gas natural está compuesta por una gran cantidad de trabajos motivados principalmente por los cambios en el precio de los combustibles, el uso eficiente de la energía y la reducción de los gases de efecto invernadero. En términos generales, se pueden clasificar según utilicen datos agregados y a nivel de hogar.

Entre los trabajos que utilizan datos agregados, Lee y Chiu (2011) estudian la demanda residencial de electricidad en 24 países de la OCDE mediante un modelo llamado panel smooth transition. Este artículo muestra que, con el tiempo, la demanda de electricidad es menos sensible a los cambios de su precio e ingreso residencial, pero más sensible a los cambios de la temperatura. Otro trabajo destacado pertenece a Dagher (2012), quien analiza la demanda residencial de gas natural en EE.UU. utilizando un modelo de retardo distribuido autorregresivo. Este autor indica que dicha demanda es más elástica en el largo plazo e insensible a los cambios en el ingreso del hogar y demuestra que la electricidad es un bien sustitutivo del gas natural.

En relación con los trabajos que utilizan datos a nivel de hogar, estos se pueden clasificar según el modelo utilizado, sea este de una ecuación, apliquen un enfoque discreto/continuo, o bien empleen un sistema de ecuaciones. Filippini y Pachauri (2004) analizan la demanda residencial de electricidad mediante tres modelos de una ecuación, uno para cada estación climática de la India: invierno, verano y monzón. Sus resultados muestran que la elasticidad precio e ingreso de la demanda de electricidad es, en valor absoluto, menor que la unidad en las tres estaciones. Los datos también muestran que, en relación con la electricidad, el gas licuado de petróleo (GLP) es considerado como un bien complementario y que el keroseno no afecta a su consumo. Por otro lado, Alberini et al. (2011) estudian la demanda residencial de electricidad y gas en EE.UU. mediante un modelo de una ecuación estático y otro dinámico. Entre sus resultados se destaca que los hogares tienen elasticidades precio de la demanda de electricidad y gas natural inelásticas tanto en el corto como en el largo plazo.

Los modelos que utilizan el enfoque discreto/continuo suponen que un individuo, familia o firma realizan, en primer lugar, una elección discreta para seleccionar qué bien consumir y después, como segundo paso, realizan una 
elección continua para decidir el consumo del bien seleccionado. Los exponentes más importantes en esta área son Dubin y McFadden (1984) y Heckman (1976, 1979). En esta línea, Vaage (2000) y Nesbakken (2001) analizan la demanda residencial de energía condicionada a la elección de los combustibles utilizados en calefacción en Noruega. En relación con sus resultados, Vaage (2000) concluye que un aumento del precio de la energía afecta negativamente las diversas opciones de calefacción y su posterior consumo. Además, un aumento del ingreso solo afecta positivamente la probabilidad de escoger una calefacción eléctrica y no la demanda de energía. Por otro lado, Nesbakken (2001) muestra que si el precio de la electricidad aumenta, dicho combustible es reemplazado por otras fuentes de energía solo en los hogares cuya tecnología se lo permite.

Con relación a los modelos de sistemas de ecuaciones, la atención está en aquellos trabajos que aplican el modelo QUAIDS, ya que es el mismo modelo utilizado en este artículo. Así, Blundell y Robin (1999) destacan por presentar un estimador lineal de mínimos cuadrados iterados (ILLE, del inglés, Iterated Linear Least Squares Estimator). Utilizando datos del Reino Unido, Blundell y Robin (1999) aplican ILLE en dos sistemas de demanda residencial concluyendo que, en comparación con el modelo LA-AIDS (del inglés, Linear Approximate Almost Ideal Demand System), la distribución de las elasticidades gasto del modelo QUAIDS es más dispersa debido a la presencia de un término cuadrático del gasto residencial. Por otro lado, el trabajo de Labandeira et al. (2006) destaca por examinar el gasto residencial español en alimentos, electricidad, gas natural, GLP, combustibles líquidos, transporte público, combustible de automóviles y otros bienes mediante un modelo QUAIDS. Entre sus resultados destaca que las elasticidades precio de los bienes del sistema son inelásticas, excepto el gas natural cuyo consumo prácticamente no cambia ante las variaciones de su precio. Además, estos autores concluyen que los hogares con menor poder adquisitivo tienen una demanda energética más elástica debido a que dicho gasto puede representar gran parte de su renta.

En relación con el consumo residencial de agua potable, el artículo de Arbúes et al. (2003) presenta una revisión de literatura acerca de este tema. Entre sus conclusiones destaca que la demanda de dicho servicio es inelástica y propone que en el futuro la demanda de agua potable se pueda analizar mediante un enfoque discreto/continuo, en donde la elección discreta del hogar sea la selección de la tarifa del servicio. Los trabajos de Martínez-Espiñeira $(2002,2007)$ destacan por examinar la demanda residencial de agua potable en Sevilla y en la zona noroeste de España, respectivamente. Ambos trabajos estiman una elasticidad precio de demanda de agua potable menor a la unidad, en términos absolutos. Según Martínez-Espiñeira (2002), esta medida de sensibilidad aumentaría durante los meses de verano y entre los hogares de mayor consumo de agua potable. También en España, esta vez con datos de Zaragoza, Arbúes et al. (2004) concluyen que la baja elasticidad precio de la demanda de agua potable se debe a la falta de bienes sustitutivos y porque el pago de su factura no representa una parte considerable de la renta familiar. También concluyen que el consumo de agua aumenta según el número de personas y el ingreso. Un mayor poder adquisitivo permitiría que las familias puedan comprar una mayor cantidad de electrodomésticos que consumen agua potable.

La Tabla 1 presenta las elasticidades precio e ingreso de la demanda residencial de electricidad, gas natural y agua potable. En general todos los trabajos 
TABLA 1

RESUMEN DE LITERATURA

\begin{tabular}{|c|c|c|c|c|c|}
\hline \multirow{2}{*}{ Autor } & \multirow{2}{*}{ País } & \multirow{2}{*}{\multicolumn{2}{|c|}{ Grupo }} & \multicolumn{2}{|c|}{ Elasticidad } \\
\hline & & & & Precio & Ingreso \\
\hline Lee y Chiu (2011) & OCDE & Electricidad & --- & de $-0,30$ a $-0,29$ & de 0,3 a 0,4 \\
\hline \multirow{5}{*}{$\begin{array}{l}\text { Leila Dagher } \\
\text { (2012) } \\
\text { Filipini y Pachauri } \\
\text { (2004) }\end{array}$} & \multirow[t]{2}{*}{ EE.UU. } & \multirow[t]{2}{*}{ Gas natural } & Corto plazo & $-0,09$ & $-0,24$ \\
\hline & & & Largo plazo & no sig. & no sig. \\
\hline & \multirow[t]{3}{*}{ India } & \multirow[t]{3}{*}{ Electricidad } & Invierno & $-0,42$ & 0,63 \\
\hline & & & Verano & $-0,29$ & 0,63 \\
\hline & & & Monzón & $-0,51$ & 0,60 \\
\hline \multirow{6}{*}{$\begin{array}{l}\text { Alberini et al. } \\
\text { (2011) }\end{array}$} & \multirow{6}{*}{ EE.UU. } & \multirow[t]{3}{*}{ Electricidad } & Modelo estático & de $-0,86$ a $-0,67$ & de 0,01 a 0,03 \\
\hline & & & $\begin{array}{l}\text { Mod. dinámico } \\
\text { Corto plazo }\end{array}$ & $-0,74$ & no sig. \\
\hline & & & $\begin{array}{l}\text { Mod. dinámico } \\
\text { Largo plazo }\end{array}$ & $-0,81$ & no sig. \\
\hline & & \multirow[t]{3}{*}{ Gas natural } & Modelo estático & de $-0,69$ a $-0,57$ & 0,01 \\
\hline & & & $\begin{array}{l}\text { Mod. dinámico } \\
\text { Corto plazo }\end{array}$ & $-0,57$ & no sig. \\
\hline & & & $\begin{array}{l}\text { Mod. dinámico } \\
\text { Largo plazo }\end{array}$ & $-0,65$ & no sig. \\
\hline Vagge (2000) & Noruega & Energía & --- & $-1,29$ & $-0,01$ \\
\hline \multirow{4}{*}{ Nesbaken (2001) } & \multirow[t]{4}{*}{ Noruega } & \multirow{2}{*}{\multicolumn{2}{|c|}{$\begin{array}{l}\text { Electricidad } \\
\text { Electr./petróleo }\end{array}$}} & $-0,55$ & 0,13 \\
\hline & & & & $-0,21$ & 0,05 \\
\hline & & \multicolumn{2}{|c|}{ Elect./madera } & $-0,18$ & 0,06 \\
\hline & & \multicolumn{2}{|c|}{ Elect./madera/petróleo } & $-0,15$ & 0,05 \\
\hline \multirow{4}{*}{$\begin{array}{l}\text { Blundell y Robin } \\
\text { (1999) }\end{array}$} & \multirow{4}{*}{$\begin{array}{l}\text { Gran } \\
\text { Bretaña }\end{array}$} & \multirow[t]{2}{*}{ QUAIDS } & Electricidad & de $-0,80$ a $-0,78$ & de 0,11 a 0,23 \\
\hline & & & Gas & $-0,44$ & de 0,58 a 0,64 \\
\hline & & \multirow[t]{2}{*}{ LA-AIDS } & Electricidad & de $-0,82$ a $-0,81$ & de 0,28 a 0,35 \\
\hline & & & Gas & $-0,42$ & de 0,53 a 0,74 \\
\hline \multirow{3}{*}{$\begin{array}{l}\text { Labandeira et al. } \\
\text { (2006) }\end{array}$} & \multirow[t]{3}{*}{ España } & \multirow[t]{3}{*}{ QUAIDS } & Electricidad & $-0,80$ & de 0,78 a 0,89 \\
\hline & & & Gas natural & $-0,45$ & de 0,58 a 1,02 \\
\hline & & & GLP & de $-0,42$ a $-0,32$ & de 0,33 a 0,36 \\
\hline $\begin{array}{l}\text { Arbues y Baeberan } \\
\text { (2004) }\end{array}$ & España & Agua potable & --- & $-0,05$ & 0,12 \\
\hline $\begin{array}{l}\text { Martinez-Espiñeira } \\
\text { (2006) }\end{array}$ & España & Agua potable & --- & $\begin{array}{c}\text { entre }-0,16 \mathrm{y} \\
\quad-0,12\end{array}$ & sin inf. \\
\hline \multirow{4}{*}{$\begin{array}{l}\text { Martínez-Espiñeira } \\
\text { (2006) } \\
\text { Ruijs et al. (2008) }\end{array}$} & \multirow[t]{2}{*}{ España } & \multirow[t]{2}{*}{ Agua potable } & Corto plazo & $-0,10$ & \multirow[t]{2}{*}{ sin inf. } \\
\hline & & & Largo plazo & $-0,50$ & \\
\hline & Brasil & Agua potable & Precio medio & $-0,46$ & 0,42 \\
\hline & & & Precio marginal & $-0,50$ & 0,39 \\
\hline Schleich y & Alemania & Agua potable & $\mathrm{MCO}$ & $-0,24$ & 0.36 \\
\hline $\begin{array}{l}\text { Hillenbrand } \\
\text { (2009) }\end{array}$ & & & $\begin{array}{l}\text { Variables } \\
\text { instrumentales }\end{array}$ & $-0,75$ & 0,46 \\
\hline
\end{tabular}

Nota: no significativo (no sig.); sin información (sin inf). Fuente: Elaboración propia.

apuntan a que dichos bienes tienen una demanda inelástica y son considerados como bienes de primera necesidad. En relación con los combustibles residenciales, estos resultados concuerdan con los trabajos de Madlener (1996) y Kristrom (2008) quienes realizan una rigurosa revisión de literatura. El primero de ellos se enfoca en presentar una clasificación de los distintos trabajos según los modelos utilizados. El segundo artículo enfatiza que la demanda de energía residencial no solo depende de elementos económicos sino también de otros factores culturales y psicológicos. 


\section{LA CENSURA EN LOS SISTEMAS DE DEMANDA}

Uno de los primeros en analizar los modelos de sistema de ecuaciones con censura en la variable dependiente es Amemiya (1974) y lo hace aplicando los fundamentos desarrollados por Tobin (1958) para un modelo de una ecuación con censura. Entre los trabajos que aplican este enfoque para analizar la demanda residencial en alimentos están Dong et al. (2004) y Yen et al. (2004).

Otra forma de analizar la censura en un sistema de ecuaciones está presente en el trabajo de Wales y Woodland (1983) quienes construyen un modelo aplicando las condiciones de Kuhn y Tucker (1951) a un problema de maximización de una función de utilidad aleatoria sujeta a las restricciones presupuestarias y de no negatividad en el consumo de los bienes. Más tarde, Lee y Pitt (1986) amplían este enfoque utilizando precios virtuales, los que pueden ser interpretados como precios de reserva o sombra de cada individuo.

Otros trabajos centrados en esta línea de investigación se caracterizan por estimar los coeficientes de un modelo de ecuaciones con censura mediante un procedimiento en dos etapas. Los pioneros en aplicar este enfoque son Heien y Wessells (1990), quienes en la primera etapa estiman un modelo probit para cada bien censurado y construyen un inverso del ratio de Mills que es incorporado, en la segunda etapa, como una variable explicativa adicional en las ecuaciones del sistema.

Posteriormente, Shonkwiler y Yen (1999) indican que el modelo propuesto por Heien y Wessells (1990) tiene una inconsistencia interna al demostrar que los individuos con una alta probabilidad de no elegir un determinado bien tienen un valor esperado incondicional de dicho consumo distinto de cero. Para tratar la censura en un sistema de ecuaciones, el enfoque de Shonkwiler y Yen (1999) multiplica la parte determinista de cada ecuación por la probabilidad de que un individuo seleccione el bien e incorporan el respectivo valor de la función de densidad como una variable explicativa adicional.

Si bien el enfoque de Shonkwiler y Yen (1999) proporciona estimadores consistentes de los parámetros del modelo, ya sea por máxima verosimilitud o mediante un sistema de ecuaciones aparentemente no relacionadas (SURE, del inglés seemingly unrelated regression equations), la estimación de las varianzas puede presentar sesgo. Al respecto, Bilgic y Yen (2013) y Zheng y Henneberry $(2010,2012)$ proponen resolver este inconveniente mediante bootstrap.

Entre los numerosos trabajos que aplican el enfoque de Shonkwiler y Yen (1999) se puede mencionar Atuesta y Paredes (2012), quienes estiman el índice espacial del coste de vida mediante un modelo AIDS aplicado a 23 principales ciudades de Colombia. Utilizando información relativa al gasto residencial de 284 tipos de alimentos, varios de ellos no consumidos en todos los hogares, muestran que existe una gran heterogeneidad del mencionado índice entre las ciudades estudiadas.

Otra aplicación del enfoque de Shonkwiler y Yen (1999), muy próximo a nuestro trabajo por utilizar datos españoles, está presente en Lasarte et al. (2014). Este artículo analiza las elasticidades de precio y de gasto de los alimentos en España mediante un modelo AIDS basado en datos de la EPF de 2010. Al igual que en el trabajo de Atuesta y Paredes (2012), la censura surge porque no todos los hogares consumen todos los alimentos. Lasarte et al. (2014) indican que las diferencias entre diferentes patrones de consumo provienen principalmente de 
los diversos ingresos familiares. Sin embargo, sus resultados también muestran la existencia de un claro efecto tamaño de ciudad: la sensibilidad a los precios de los hogares en ciudades grandes es mayor que la de aquellas familias ubicadas en ciudades pequeñas y en zonas rurales.

Otro artículo destacado en el campo de los modelos censurados pertenece a Tauchmann (2005), quien analiza un modelo de sistemas de ecuaciones con censura aplicando los conceptos de Heckman (1976, 1979). De este modo, a diferencia de los enfoques anteriores, en la segunda etapa del proceso de estimación solo se emplean las observaciones donde se observa el consumo. En concreto, Tauchmann (2005) demuestra que la incorporación del inverso del ratio de Mills en cada ecuación del sistema proporciona estimadores de los coeficientes cuya consistencia depende de la forma que tiene la matriz de varianzas y covarianzas de los errores del modelo.

Más tarde, con el objeto de obtener estimadores consistentes y con menor error cuadrático medio, Tauchmann (2010) reformula su anterior proceso de estimación de tal forma que, en la primera etapa, se estima un modelo probit multivariante sobre todos los bienes censurados y, con esta información, se construye un término de corrección generalizado basado en los principios de Heckman (1976, 1979). En la segunda etapa se incorpora dicho término generalizado en todas las ecuaciones del sistema y sus coeficientes se estiman mediante mínimos cuadrados ordinarios (MCO), SURE y por mínimos cuadrados ordinarios generalizados (MCG). Es importante destacar que con cualquiera de estos métodos se logran estimadores consistentes, sin embargo solo con MCG se consigue el menor error cuadrático medio.

Mediante la simulación, Tauchman (2010) concluye que los estimadores que surgen de la generalización multivariante del procedimiento clásico en dos etapas de Heckman $(1976,1979)$, que tiene en cuenta la correlación entre ecuaciones y utiliza el inverso ratio de Mills como término de corrección, son consistentes solamente si la matriz de varianzas y covarianzas de los errores del sistema cumple ciertas condiciones. Con el objeto de obtener estimadores consistentes bajo condiciones más generales, Tauchmann (2010) generaliza el procedimiento clásico de dos etapas, basándose en el patrón de selección conjunta de bienes en el sistema de ecuaciones. El procedimiento de estimación aplicado en el presente trabajo aplica esta generalización y se describe a continuación. En nuestro caso se trata de un caso específico de un sistema de demanda con un solo bien censurado.

\section{El Modelo Quaids censurado}

De acuerdo con Tauchmann (2010), un modelo de sistema de ecuaciones cuyas variables explicadas tienen censura se representa de la siguiente forma:

$$
\begin{aligned}
& y_{i t}^{*}=x_{i t}^{\prime} \beta_{i}+\varepsilon_{i t} \\
& d_{i t}^{*}=z_{i t}^{\prime} \pi_{i}+v_{i t},
\end{aligned}
$$

donde el subíndice $i=1, \ldots, m$ identifica al $i$-ésimo bien de un total de $m$ bienes que corresponden a las ecuaciones del sistema de demanda y $t=1, \ldots, T$ se refiere 
al $t$-ésimo individuo de la muestra aleatoria de tamaño $T$. Los términos del lado izquierdo, $y^{*}$ y $d^{*}$, son variables latentes que caracterizan las decisiones de consumo y la elección del bien, respectivamente. En el lado derecho de ambas ecuaciones, los vectores $x_{i t}^{\prime} \mathrm{y} z_{i t}^{\prime}$ representan las variables explicativas observadas junto con sus respectivos vectores de coeficientes $\beta_{i}$ y $\pi_{i}$. Los términos de error $\varepsilon_{i t}$ y $v_{i t}$ son variables aleatorias normalmente distribuidas con media cero y con la siguiente matriz de varianzas y covarianzas:

$$
\operatorname{Var}\left(\varepsilon_{i t}, v_{i t}\right)=\left[\begin{array}{cc}
\Sigma_{\varepsilon \varepsilon} & \Sigma_{\varepsilon v}^{\prime} \\
\Sigma_{\varepsilon v} & \Sigma_{v v}
\end{array}\right]
$$

donde los elementos de la diagonal de $\Sigma_{v v}$ están sujetos a normalización. De este modo, $\sigma_{i i}^{v v}=1, i=1, \ldots, m$. El modelo determina que el consumo $y_{i t}$ se observa para un individuo $t$, siempre que $d^{*}>0$, es decir:

$$
\begin{gathered}
d_{i t}= \begin{cases}1 & \text { si } d_{i t}^{*}>0 \\
0 & \text { si } d_{i t}^{*} \leq 0\end{cases} \\
y_{i t}=d_{i t} y_{i t}^{*} .
\end{gathered}
$$

De este modo, el modelo de Tauchmann (2010) es el siguiente:

$$
y_{i t}=d_{i t} x_{i t}^{\prime} \beta_{i}+d_{i t} \sum_{j=1}^{m} \delta_{i j} \psi_{j t} \phi\left(z_{j t}^{\prime} \pi_{j}\right) \frac{\Phi^{m-1}\left(\tilde{A}_{j t}, \tilde{R}_{j t}\right)}{\Phi^{m}(\cdot)}+d_{i t} \tilde{\varepsilon}_{j t},
$$

donde $\delta_{i j}$ son los coeficientes del término de corrección generalizado. La variable $\psi_{j t}$ se define como $2 d_{j t}-1$, el término $\phi$ corresponde a la función de densidad de probabilidad univariante normal estándar y $\Phi^{m}$ denota la función de distribución acumulada de una distribución $m$-variante normal estándar. El vector $\tilde{A}_{j t}$ contiene $m-1$ elementos y se define de la siguiente manera:

$$
\tilde{A}_{j t}=\frac{\psi_{l t}\left(z_{l t}^{\prime} \pi_{l}-\sigma_{l j}^{v v} z_{j t}^{\prime} \pi_{j}\right)}{\left(1-\left(\sigma_{l j}^{v v}\right)^{2}\right)^{1 / 2}}, \quad l=1, \ldots, m, \quad l \neq j,
$$

donde $\sigma_{l j}^{v v}$ corresponde al elemento de la $l$-ésima fila y j-ésima columna de $\Sigma_{v v}$. Por otro lado, $\tilde{R}_{j t}$ se define como:

$$
\tilde{R}_{j t}=\Psi_{j t} R_{j t} \Psi_{j t}
$$

donde $R_{j t}$ representa la matriz de correlación parcial condicional $\operatorname{Cor}\left(v_{t} \mid v_{j t}\right)$ y $\psi_{j t}$ denota una matriz diagonal cuyos elementos son $\psi_{l t}$ con $l \neq t$. El término $\Phi^{m}(\cdot)$ corresponde a la probabilidad conjunta de observar el patrón $d_{t}$, es decir, $\left(d_{1}, d_{2 t}, \ldots, d_{m t}\right)$. Finalmente, $\tilde{\varepsilon}_{i t}$ es el error aleatorio que afecta el consumo de cada i-ésimo bien. 
A continuación se indica cómo el término de corrección generalizado se adapta para analizar un sistema de ecuaciones compuesto por $m=3$ bienes. De (5) se puede demostrar que dicho término se desglosa en tres variables que afectan a cada ecuación del sistema de la siguiente forma:

$$
\begin{aligned}
& y_{1 t}=d_{1 t} x_{1 t}^{\prime} \beta_{1}+d_{1 t}\left(\delta_{11} M_{1 t}+\delta_{12} M_{2 t}+\delta_{13} M_{3 t}\right)+d_{1 t} \tilde{\varepsilon}_{1 t} \\
& y_{2 t}=d_{2 t} x_{2 t}^{\prime} \beta_{2}+d_{2 t}\left(\delta_{21} M_{1 t}+\delta_{22} M_{2 t}+\delta_{23} M_{3 t}\right)+d_{2 t} \tilde{\varepsilon}_{2 t} \\
& y_{3 t}=d_{3 t} x_{3 t}^{\prime} \beta_{3}+d_{3 t}\left(\delta_{31} M_{1 t}+\delta_{32} M_{2 t}+\delta_{33} M_{3 t}\right)+d_{3 t} \tilde{\varepsilon}_{3 t},
\end{aligned}
$$

donde

$$
\begin{gathered}
M_{1 t}=\psi_{1 t} \phi\left(z_{1 t}^{\prime} \pi_{1}\right) \frac{\Phi^{2}\left(\frac{\psi_{2 t}\left(z_{2 t}^{\prime} \pi_{2}-\sigma_{21}^{v v} z_{1 t}^{\prime} \pi_{1}\right)}{\left(1-\left(\sigma_{21}^{v v}\right)^{2}\right)^{1 / 2}}, \frac{\psi_{3 t}\left(z_{3 t}^{\prime} \pi_{3}-\sigma_{31}^{v v} z_{1 t}^{\prime} \pi_{1}\right)}{\left(1-\left(\sigma_{31}^{v v}\right)^{2}\right)^{1 / 2}}\right)}{\Phi^{3}\left(z_{1 t}^{\prime} \pi_{1}, z_{2 t}^{\prime} \pi_{2}, z_{3 t}^{\prime} \pi_{3}\right)} \\
\left.M_{2 t}=\psi_{2 t} \phi\left(z_{2 t}^{\prime} \pi_{2}\right) \frac{\Phi^{2}\left(\frac{\psi_{1 t}\left(z_{1 t}^{\prime} \pi_{1}-\sigma_{12}^{v v} z_{2 t}^{\prime} \pi_{2}\right)}{\left(1-\left(\sigma_{12}^{v v}\right)^{2}\right)^{1 / 2}}, \frac{\psi_{3 t}\left(z_{3 t}^{\prime} \pi_{3}-\sigma_{32}^{v v} z_{2 t}^{\prime} \pi_{2}\right)}{\left(1-\left(\sigma_{32}^{v v}\right)^{2}\right)^{1 / 2}}\right)}{\Phi^{3}\left(z_{1 t}^{\prime} \pi_{1}, z_{2 t}^{\prime} \pi_{2}, z_{3 t}^{\prime} \pi_{3}\right)}\right) \\
M_{3 t}=\psi_{3 t} \phi\left(z_{3 t}^{\prime} \pi_{3}\right) \frac{\Phi^{2}\left(\frac{\psi_{1 t}\left(z_{1 t}^{\prime} \pi_{1}-\sigma_{13}^{v v} z_{3 t}^{\prime} \pi_{3}\right)}{\left(1-\left(\sigma_{13}^{v v}\right)^{2}\right)^{1 / 2}}, \frac{\psi_{2 t}\left(z_{2 t}^{\prime} \pi_{2}-\sigma_{23}^{v v} z_{3 t}^{\prime} \pi_{3}\right)}{\left(1-\left(\sigma_{23}^{v v}\right)^{2}\right)^{1 / 2}}\right)}{\Phi^{3}\left(z_{1 t}^{\prime} \pi_{1}, z_{2 t}^{\prime} \pi_{2}, z_{3 t}^{\prime} \pi_{3}\right)},
\end{gathered}
$$

Si se supone que los dos primeros bienes del sistema ( $i=1$ y 2 ) corresponden a la electricidad y el agua potable, y el tercer bien $(i=3)$ representa el gas natural, las expresiones (1) y (2) se mantienen, pero las reglas de decisión en (3) y (4) cambian del siguiente modo:

$$
\begin{gathered}
d_{1 t}=d_{2 t}=1 \\
d_{3 t}=\left\{\begin{array}{cc}
1 & \text { si } t=1,2, \ldots, r>0 \\
0 & \text { si } t=r+1, \ldots, T>0
\end{array},\right.
\end{gathered}
$$

donde el consumo del tercer bien se observa solo en las primeras $r$ observaciones. De acuerdo con Shonkwiler y Yen (1999), el cumplimiento de (6) y (7) implica que $z_{1 t}^{\prime} \pi_{1} \rightarrow+\infty, z_{2 t}^{\prime} \pi_{2} \rightarrow+\infty$ y $z_{3 t} \pi_{3}$ sea un número finito. Esto trae como consecuencia que:

$$
\lim _{z_{1 t}^{\prime} \pi_{1} \rightarrow+\infty} \phi\left(z_{1 t}^{\prime} \pi_{1}\right)=\lim _{z_{2 t}^{\prime} \pi_{2} \rightarrow+\infty} \phi\left(z_{2 t}^{\prime} \pi_{2}\right)=0
$$

Además, si se consideran las propiedades de una función de distribución acumulada multivariante (Ibe, 2005, pp. 168) se tiene que: 


$$
0<\lim _{\substack{z_{1 t}^{\prime} \pi_{1} \rightarrow+\infty \\ z_{2 t}^{\prime} \pi_{2} \rightarrow+\infty}} \Phi^{3}\left(z_{1 t}^{\prime} \pi_{1}, z_{2 t}^{\prime} \pi_{2}, z_{3 t}^{\prime} \pi_{3}\right)=\Phi\left(z_{3 t}^{\prime} \pi_{3}\right)<1
$$

Por lo tanto, si se tiene en cuenta que $\psi_{1 t}=\psi_{2 t}=1$, el cumplimiento de (6) conlleva que las variables $M_{1 t}$ y $M_{2 t}$ tienden a cero. Por las mismas razones señaladas los valores correspondientes a cada $\Phi^{2}(\cdot)$ en las mencionadas variables son diferentes de cero.

Además, el cumplimiento de (6) y (7) implica que $M_{3 t}$ se transforme en un inverso del ratio de Mills cuya información se extrae a partir de un modelo probit univariante del bien censurado. Esto se debe al resultado presente en (8), al valor unitario que asume $\psi_{3 t}$ y al valor de la función de distribución acumulada bivariante que tiende a la unidad a medida que sus argumentos tienden al infinito. En consecuencia, considerando todo lo anterior, el cumplimiento de (6) y (7) transforma el sistema de ecuaciones definido en (5) en:

$$
y_{i t}=d_{i t} x_{i t}^{\prime} \beta_{i}+d_{i t} \delta_{i 3}\left[\frac{\phi\left(z_{3 t}^{\prime} \pi_{3}\right)}{\Phi\left(z_{3 t}^{\prime} \pi_{3}\right)}\right]+d_{i t} \tilde{\varepsilon}_{i t}
$$

Ahora se incorpora el resultado de este análisis en el modelo econométrico utilizado en este artículo. Tal como se ha mencionado, la demanda residencial de electricidad, agua potable y gas natural se analiza mediante el modelo QUAIDS propuesto por Banks et al. (1997), que anida al modelo AIDS (del inglés, Almost Ideal Demand System) de Deaton y Muellbauer (1980). Es esencial mencionar que ambos modelos tienen una forma funcional que refleja la conducta racional del consumidor.

En este artículo el modelo QUAIDS original es adaptado para incorporar la información sociodemográfica y para tratar la posible endogeneidad del gasto residencial total. Las características de cada hogar $\left(\right.$ socdem $\left._{k}\right)$ se agregan a cada ecuación del sistema en forma lineal de acuerdo con Pollak y Wales (1992). Por otro lado, la probable endogeneidad del gasto total, que surge de la correlación de dicha variable con el término de error aleatorio presente en cada ecuación, se trata siguiendo el enfoque Blundell y Robin (1999): en primer lugar se realiza una regresión que relaciona el gasto total en el sistema de demanda con todas las variables independientes del modelo más el ingreso que actúa como variable instrumental. En segundo lugar, los residuos de esta regresión $(\hat{\epsilon})$ se incorporan a cada ecuación del sistema como una variable independiente adicional y se estima el modelo. Además de Blundell y Robin (1999), este tratamiento se aplica en Labandeira et al. (2006) y Zheng y Henneberry (2010).

De este modo, omitiendo el subíndice $t$ por simplicidad, el modelo utilizado es el siguiente:

$$
\begin{aligned}
w_{i}=d_{i} \alpha_{i} & +d_{i} \sum_{j} \gamma_{i j} \ln p_{j}+d_{i} \beta_{i} \ln \left(\frac{x}{P}\right)+d_{i} \frac{\lambda_{i}}{b(p)}\left[\ln \left(\frac{x}{P}\right)\right]^{2} \\
& +d_{i} \sum_{k} \theta_{i k} \operatorname{socdem}_{k}+d_{i} \zeta_{i} \hat{\epsilon}+d_{i} \delta_{i 3}\left[\frac{\phi\left(z_{3}^{\prime} \pi_{3}\right)}{\Phi\left(z_{3}^{\prime} \pi_{3}\right)}\right]+d_{i} \tilde{\varepsilon}_{i}
\end{aligned}
$$


donde $w_{i}$ es cuota de gasto residencial en electricidad, agua potable y gas natural. Los precios y el gasto total se representan por $p_{i}$ y $x$, respectivamente. Los términos $\alpha_{i}, \gamma_{i j}, \beta_{i}$ y $\lambda_{i}$ son los parámetros del modelo QUAIDS original. Las $k$ variables sociodemográficas del hogar se representan por medio de ( socdem $_{k}$ ) y $\theta_{i k}$ son sus parámetros. El término $\hat{\epsilon}$ corresponde al residuo de la regresión auxiliar para tratar la endogenidad del gasto total y $\zeta_{i}$ es su parámetro. El vector $z_{3}$ contiene las variables explicativas utilizadas en modelo probit univariante que describe la elección residencial del gas natural y $\hat{\pi}_{3}$ es el vector que contiene sus parámetros estimados. La variable $\varepsilon_{i}$ representa el término de error que afecta cada ecuación del sistema. Por otro lado, los términos $P$ y $b(p)$ corresponden a los siguientes índices de precios:

$$
\begin{gathered}
\ln P=\alpha_{0}+\sum_{i} \alpha_{i} \ln p_{i}+\frac{1}{2} \sum_{i} \sum_{j} \gamma_{i j} \ln p_{i} \ln p_{j}, \\
b(p)=\prod_{i} p_{i}^{\beta_{i}}
\end{gathered}
$$

Para que el modelo refleje el comportamiento de un consumidor racional, los coeficientes del modelo QUAIDS deben cumplir con las siguientes restricciones:

$$
\begin{gathered}
\sum_{i} \alpha_{i}=1, \quad \sum_{i} \gamma_{i j}=\sum_{i} \beta_{i}=\sum_{i} \theta_{i k}=\sum_{i} \zeta_{i}=\sum_{i} \delta_{i 3}=\sum_{i} \lambda_{i}=0 . \\
\sum_{j} \gamma_{i j}=0 \\
\gamma_{i j}=\gamma_{j i}
\end{gathered}
$$

donde (10) se conoce como condición de agregación: todas las cuotas de gasto del sistema de ecuaciones deben sumar $100 \%$. Es conveniente destacar que la modificación del modelo QUAIDS original no altera el cumplimiento de esta condición, ya que todas las ecuaciones tienen las mismas variables explicativas. La igualdades en (11) y (12) se llaman condición de homogeneidad y simetría, respectivamente.

Un aspecto clave a considerar en la estimación de cualquier modelo QUAIDS es el valor de parámetro $\alpha_{0}$ presente en (9). De acuerdo con Deaton y Muellbauer (1980), este parámetro no puede ser estimado junto con el resto de los coeficientes del modelo y, por tanto, debe ser conocido de antemano. En este artículo seguimos la propuesta de Holt y Goodwin (2009) y utilizamos aquel valor de $\alpha_{0}$ que maximiza la función de verosimilitud del modelo. Además, para evitar la singularidad de la matriz de varianzas y covarianzas del modelo QUAIDS, una de las ecuaciones no sea considerada dentro del proceso de estimación (Labandeira et al., 2006). En este caso, siguiendo el criterio de Zheng y Henneberry (2010), el gasto residencial en agua potable queda excluido por tener la menor cuota de gasto entre los bienes analizados.

Los coeficientes del modelo QUAIDS se obtienen mediante el método de mínimos cuadrados generalizados factibles no lineal e iterativo (IFGNLS, por sus siglas en inglés). Este método considera que las ecuaciones del sistema 
están aparentemente no relacionadas y además es equivalente a la aplicación del método de máxima verosimilitud (Poi, 2008). Esta característica permite determinar el valor adecuado del parámetro $\alpha_{0}$.

\section{Datos}

Los datos del sistema de demanda censurado pertenecen a la EPF de 2012, que es la fuente de información más completa disponible sobre el gasto de los hogares españoles y sus características sociodemográficas. Este instrumento se viene aplicando desde 2006 en alrededor de 24 mil hogares. La EPF contiene información sobre el gasto y la cantidad consumida en electricidad, gas natural y agua potable para cada hogar de la muestra.

Con el propósito de eliminar las observaciones atípicas, la muestra no considera los hogares que no consumen electricidad o agua potable, ya que corresponden a casas desocupadas. También fueron eliminados aquellos hogares que no disponen de información sobre alguna de las variables del estudio; los hogares cuyo gasto en cada combustible residencial es inferior a $10 € /$ año y aquellas familias cuyos precios de la energía estuviesen bajo y sobre los percentiles 2 y 98 , respectivamente. Para mantener cierta homogeneidad en el acceso a la energía solo se consideran los hogares ubicados en la zona peninsular de España. Este procedimiento redujo el tamaño de la muestra, en una primera fase, a 12.776 hogares.

La Tabla 2 describe la combinación de fuentes energéticas utilizadas por los hogares de la muestra. Los datos señalan que la combinación de combustibles predominante es la electricidad y el gas natural. Debido a que la atención está en el uso de los principales combustibles residenciales, la muestra se compone de 9.655 observaciones. De ella se observa que el 44,7\% de los hogares no utiliza gas natural. Por tanto, mientras que el consumo de electricidad y agua potable está presente en toda la muestra, el consumo de gas natural es cero para una gran cantidad de hogares dando origen a la censura.

Con la información disponible en la EPF se construye un precio para cada bien dividiendo el gasto entre su correspondiente cantidad consumida, del mismo modo que Gundimeda y Köhlin (2008), Zheng y Henneberry (2012) y Bilgic y Yen (2013). En los hogares donde no se observa el consumo de gas natural u otro combustible residencial, el precio de este combustible se obtiene a partir de la predicción de un modelo auxiliar que regresa el precio de los hogares, donde se conoce esta información, sobre un conjunto de variables sociodemográficas (Heien y Wessells, 1990; Hoderlein y Mihaleva, 2008).

La Tabla 3 presenta las variables del estudio junto con sus principales estadísticos descriptivos para los hogares que consumen todos los bienes. Esto se debe a que el enfoque de Tauchmann (2010) solo utiliza estos hogares en la segunda etapa de estimación del modelo. En ella los hogares aparecen agrupados según su ubicación geográfica del siguiente modo: zona central (Castilla y León, Castilla-La Mancha y Extremadura), zona este (Cataluña y Comunitat Valenciana), Madrid, zona noreste (Aragón, La Rioja, Navarra, País Vasco), zona noroeste (Asturias, Cantabria, Galicia) y zona sur (Andalucía y Murcia).

La muestra indica que, en promedio, el consumo de electricidad representa la mitad del gasto residencial y que, sumado al gasto en gas natural, esta cifra se eleva al $88 \%$. Según los datos, en promedio, cada hogar está formado por 
TABLA 2

NÚMERO DE HOGARES SEGÚN COMBINACIÓN DE COMBUSTIBLES

RESIDENCIALES

\begin{tabular}{|lcc|}
\hline Combustibles & Número de hogares & Porcentaje \\
\hline Electricidad y gas natural & 5.335 & 41,80 \\
Solo electricidad & 4.320 & 33,80 \\
Electricidad y gasóleo & 1.671 & 13,10 \\
Electricidad y GLP & 1.125 & 8,80 \\
Electricidad y dos combustibles & 270 & 2,10 \\
Electricidad y tres combustibles & 55 & 0,40 \\
Total & 12.776 & 100,00 \\
\hline
\end{tabular}

Fuente: Elaboración propia.

TABLA 3

VARIABLES DEL MODELO QUAIDS CON CENSURA Y SUS ESTADÍSTICOS DESCRIPTIVOS

\begin{tabular}{|lrrrr|}
\hline Variable & Promedio & Desviación & Mín. & Máx. \\
\hline Cuota de gasto residencial en: & & & & \\
Electricidad & 0,5 & 0,156 & 0,043 & 0,94 \\
Gas natural & 0,38 & 0,17 & 0,016 & 0,892 \\
Agua potable & 0,121 & 0,075 & 0,008 & 0,654 \\
Variables explicativas: & & & & \\
Precio electricidad $(€ / \mathrm{kwh})$ & 0,236 & 0,034 & 0,14 & 0,352 \\
Precio gas natural $\left(€ / \mathrm{m}^{3}\right)$ & 1,231 & 0,531 & 0,187 & 3,377 \\
Precio agua potable $\left(€ / \mathrm{m}^{3}\right)$ & 1,005 & 0,022 & 0,933 & 1,079 \\
Gasto total (miles de $€ /$ año) & 1,422 & 0,694 & 0,159 & 6,724 \\
Ingreso del hogar (miles de $€ /$ mes) & 2,238 & 1,254 & 0,25 & 1,139 \\
Número de personas & 2,701 & 1,184 & 1 & 12 \\
Sustentador principal con educación & 0,1 & 0,3 & 0 & 1 \\
básica & & & & \\
Sustentador principal con educación & 0,512 & 0,5 & 0 & 1 \\
secundaria & & & & \\
Sustentador principal con educación & 0,388 & 0,487 & 0 & 1 \\
superior & & & & \\
Sustentador principal mujer & 0,297 & 0,457 & 0 & 1 \\
Sustentador principal jubilado & 0,242 & 0,428 & 0 & 1 \\
Vivienda en alquiler & 0,548 & 0,498 & 0 & 1 \\
Superficie (m²) & 94,864 & 39,388 & 35 & 300 \\
Piso & 0,884 & 0,321 & 0 & 1 \\
Zona central & 0,095 & 0,294 & 0 & 1 \\
Zona este & 0,27 & 0,444 & 0 & 1 \\
Zona madrid & 0,143 & 0,35 & 0 & 1 \\
Zona noreste & 0,327 & 0,469 & 0 & 1 \\
Zona noroeste & 0,121 & 0,326 & 0 & 1 \\
Zona sur & 0,044 & 0,205 & 0 & 1 \\
\hline
\end{tabular}

Nota: La variable gasto total corresponde a la suma del gasto residencial el electricidad, gas natural y agua potable. Fuente: Elaboración propia. 
alrededor de 3 personas, tiene un ingreso de $2.238 € /$ mes y gasta en electricidad, agua potable y gas natural una media de $1.422 € /$ año, o bien $118,5 € /$ mes. Por otro lado, gran parte de los sustentadores principales tienen una educación distinta de la básica. De hecho, solo el 10\% de ellos alcanza el nivel más bajo de formación. Además, los datos muestran que el género de los sustentadores principales es predominantemente masculino. Respecto de su situación laboral el 24,2\% de ellos está jubilado. Respecto de la vivienda, el 54,8\% se encuentra en alquiler. Cada una tiene un promedio de $94,9 \mathrm{~m}^{2}$ y el $88,4 \%$ es un piso o departamento.

\section{Resultados}

Esta sección presenta los resultados más importantes de la estimación del modelo QUAIDS para la demanda residencial de electricidad, gas natural y agua potable. La Tabla 4 presenta las estimaciones del modelo probit obtenidas mediante el método de máxima verosimilitud. Este modelo determina la probabilidad de observar el gasto residencial en gas natural en un hogar de la muestra.

Los resultados del modelo probit indican que los precios de la electricidad y agua potable tienen una relación positiva con la probabilidad de que un hogar consuma gas natural. Como es de esperar, un aumento en el precio del gas natural disminuye la probabilidad de observar su consumo en un hogar. En relación con las variables sociodemográficas, la probabilidad de tener gas natural en un hogar aumenta si el sustentador principal está jubilado, tiene una educación distinta de la básica, o bien si la familia habita una vivienda en un bloque de departamentos. Las viviendas ubicadas en la zona central, este, noroeste y sur del país tienen menos probabilidad de utilizar el gas natural respecto de aquellas ubicadas en Madrid y en la zona noroeste.

La Tabla 5 presenta el modelo de regresión auxiliar estimado mediante $\mathrm{MCO}$ para tratar la posible endogeneidad del gasto residencial en electricidad, gas natural y agua potable en el modelo QUAIDS. Los resultados indican que el coeficiente de la variable ln(ingreso), que es la variable instrumental utilizada en la literatura, es significativa y además tiene el signo esperado. En consecuencia, cuanto mayor es el ingreso del hogar, mayor es el gasto residencial en los bienes analizados.

De acuerdo con lo mencionado, el valor de $\alpha_{0}$ en el índice de precios (9) se obtiene calculando el máximo de la función de verosimilitud del modelo para diferentes valores de este. En la Figura 1 se aprecia que dicho máximo se encuentra cuando $\alpha_{0}=9,3$.

Los coeficientes estimados del modelo QUAIDS con censura están en la Tabla 6. Los datos muestran que los precios de la electricidad, gas natural y agua potable son significativos en sus respectivas ecuaciones. En general, las variables explicativas son significativas en al menos dos de los tres bienes del modelo. La Tabla 6 también muestra que los coeficientes asociados al gasto residencial, lineal y cuadrático, son significativos. Esto confirma la idoneidad del modelo QUAIDS respecto del modelo AIDS, a pesar de que los coeficientes que acompañan al término cuadrático del gasto residencial en las tres ecuaciones son pequeños. Además, la significatividad de los residuos de la regresión auxiliar confirma que el gasto residencial en electricidad, gas natural y agua potable es una variable endógena. La significatividad del inverso del ratio de 
TABLA 4

MODELO PROBIT PARA LA PRESENCIA DE GAS NATURAL EN LA MUESTRA

\begin{tabular}{|llc|}
\hline $\begin{array}{l}\text { Variable dependiente: } \\
\mathrm{y}_{\mathrm{t}}=1 \text { si la vivienda tiene gas natural } \\
\mathrm{y}_{\mathrm{t}}=0 \text { si la vivienda no tiene gas natural }\end{array}$ & \\
\hline Variables explicativas: & & \\
\hline Constante & Coeficiente & Valor-p \\
ln (precio electricidad) & $6,478^{* * *}$ & $(0,000)$ \\
ln (precio gas natural) & $1,060^{* * *}$ & $(0,000)$ \\
ln (precio agua potable) & $0,431^{* * *}$ & $(0,000)$ \\
Número de personas & $0,241^{* * *}$ & $(0,000)$ \\
Sustentador principal con educación secundaria & $0,070^{* * *}$ & $(0,000)$ \\
Sustentador principal con educación superior & $0,289^{* * *}$ & $(0,000)$ \\
Sustentador principal mujer & $0,561^{* * *}$ & $(0,000)$ \\
Sustentador principal jubilado & 0,050 & $(0,152)$ \\
Vivienda en alquiler & $0,076^{*}$ & $(0,061)$ \\
Superficie & 0,04 & $(0,240)$ \\
Piso & $0,001^{* * *}$ & $(0,002)$ \\
Zona central & $1,016^{* * *}$ & $(0,000)$ \\
Zona este & $0,345^{* * *}$ & $(0,000)$ \\
Zona noreste & $0,375^{* * *}$ & $(0,000)$ \\
Zona noroeste & $0,286^{* * *}$ & $(0,000)$ \\
Zona sur & $0,182^{* * *}$ & $(0,012)$ \\
ln (gasto total) & $1,501^{* * *}$ & $(0,000)$ \\
Observaciones & $1,064^{* * *}$ & $(0,000)$ \\
Pseudo R & 9.655 & \\
\hline
\end{tabular}

Nota: $*(\mathrm{p}<0,10), * *(\mathrm{p}<0,05), * * *(\mathrm{p}<0,01)$.

Fuente: Elaboración propia.

Mills también corrobora la importancia del tratamiento de la censura del gasto residencial en gas natural.

La Tabla 7 presenta las elasticidades precio, compensadas y no compensadas, gasto e ingreso de la demanda residencial de electricidad, gas natural y agua potable computadas para un hogar medio de la muestra (en adelante, cuando se lea elasticidad precio de la demanda, este trabajo se refiere a la elasticidad precio de la demanda no compensada) junto con sus intervalos de confianza calculados mediante el método delta. Los resultados muestran que la electricidad y el agua potable tienen una demanda inelástica. El gas natural tiene una elasticidad precio que, en valor absoluto, supera la unidad y casi duplica las de la electricidad y el agua potable. Esto indica que los hogares son notablemente más sensibles a los cambios en el precio del gas natural.

Como se observa, la distribución de las elasticidades precio del gas natural tienen el intervalo de confianza más ancho, mientras que el agua potable tiene el intervalo más estrecho. En el caso de la electricidad, considerando un nivel de significatividad del 5\%, los resultados rechazarían la hipótesis de una elasticidad precio de la demanda unitaria, o bien elástica. 
TABLA 5

REGRESIÓN AUXILIAR DEL GASTO RESIDENCIAL TOTAL

Variable dependiente:

$\ln$ (gasto total)

Variables explicativas:

Constante

$\ln$ (precio electricidad)

$\ln$ (precio gas natural)

$\ln$ (precio agua potable)

Número de personas

Sustentador principal con educación secundaria

Sustentador principal con educación superior

Sustentador principal mujer

Sustentador principal jubilado

Vivienda en alquiler

Superficie

Piso

Zona central

Zona este

Zona noreste

Zona noroeste

Zona sur

ln (ingreso)

Observaciones

$\mathrm{R}^{2}$

\begin{tabular}{cc} 
Coeficiente & Valor-p \\
\hline $4,722 * * *$ & $(0,000)$ \\
$-0,261 * * *$ & $(0,000)$ \\
$-0,189 * * *$ & $(0,000)$ \\
0,012 & $(0,359)$ \\
$0,097 * * *$ & $(0,000)$ \\
$0,114 * * *$ & $(0,000)$ \\
$0,113 * * *$ & $(0,000)$ \\
0,008 & $(0,486)$ \\
$-0,002$ & $(0,890)$ \\
0,009 & $(0,410)$ \\
$0,002 * * *$ & $(0,000)$ \\
$0,080 * * *$ & $(0,000)$ \\
$-0,164 * * *$ & $(0,000)$ \\
$-0,071 * * *$ & $(0,000)$ \\
$-0,121 * * *$ & $(0,000)$ \\
$-0,188 * * *$ & $(0,000)$ \\
$-0,191 * * *$ & $(0,000)$ \\
$0,188 * * *$ & $(0,000)$ \\
9.655 & \\
$24,20 \%$ &
\end{tabular}

Nota: $*(\mathrm{p}<0,10), * *(\mathrm{p}<0,05), * * *(\mathrm{p}<0,01)$.

Fuente: Elaboración propia.

FIGURA 1

VALORES DE LA FUNCIÓN DE VEROSIMILITUD DEL MODELO QUAIDS CON CENSURA

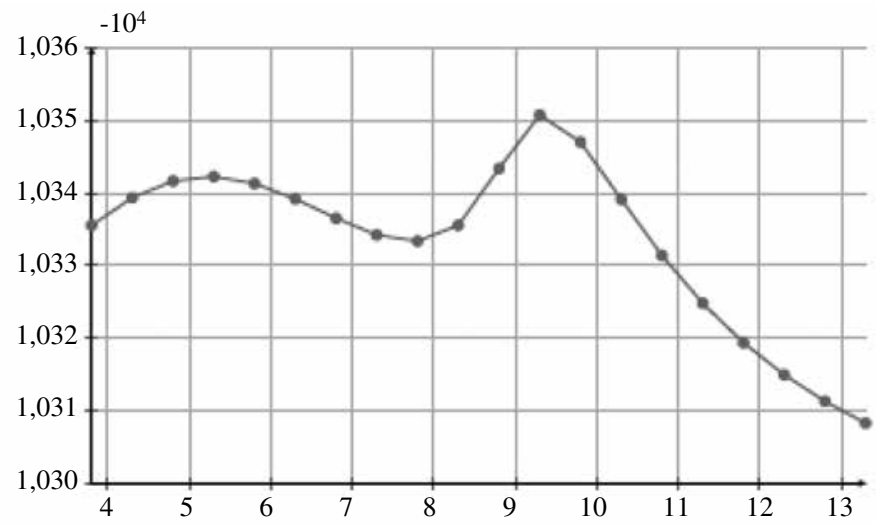

Fuente: Elaboración propia. 
TABLA 6

ESTIMACIONES DEL MODELO QUAIDS CENSURADO

\begin{tabular}{|lcccccc|}
\hline & \multicolumn{2}{c}{ Electricidad } & \multicolumn{2}{c}{ Gas natural } & \multicolumn{2}{c|}{ Agua potable } \\
\hline Variables explicativas: & Coeficiente & Valor-p & Coeficiente & Valor-p & Coeficiente & Valor-p \\
\hline Constante & 0,183 & $(0,000)$ & 0,808 & $(0,000)$ & 0,009 & $(0,570)$ \\
$\ln$ (precio electricidad) & 0,113 & $(0,000)$ & $-0,123$ & $(0,000)$ & 0,017 & $(0,000)$ \\
$\ln$ (precio gas natural) & $-0,123$ & $(0,000)$ & 0,176 & $(0,000)$ & $-0,046$ & $(0,000)$ \\
$\ln$ (precio agua potable) & 0,017 & $(0,003)$ & $-0,046$ & $(0,000)$ & 0,029 & $(0,000)$ \\
$\ln$ (gasto total) & $-0,332$ & $(0,000)$ & 0,364 & $(0,000)$ & $-0,032$ & $(0,005)$ \\
$\ln$ (gasto total) & $-0,040$ & $(0,055)$ & 0,018 & $(0,354)$ & 0,022 & $(0,039)$ \\
Número de personas & $-0,094$ & $(0,000)$ & 0,083 & $(0,000)$ & 0,010 & $(0,030)$ \\
Sustentador principal con & 0,012 & $(0,000)$ & $-0,020$ & $(0,000)$ & 0,009 & $(0,000)$ \\
educación secundaria & & & & & & \\
Sustentador principal con & 0,017 & $(0,055)$ & $-0,023$ & $(0,009)$ & 0,006 & $(0,094)$ \\
educación superior & & & & & & \\
Sustentador principal mujer & 0,005 & $(0,686)$ & $-0,013$ & $(0,264)$ & 0,008 & $(0,091)$ \\
Sustentador principal jubilado & $-0,009$ & $(0,043)$ & 0,008 & $(0,072)$ & 0,001 & $(0,552)$ \\
Vivienda en alquiler & $-0,011$ & $(0,046)$ & 0,010 & $(0,082)$ & 0,002 & $(0,483)$ \\
Superficie & $-0,008$ & $(0,068)$ & 0,008 & $(0,058)$ & 0,000 & $(0,913)$ \\
Piso & 0,000 & $(0,013)$ & 0,000 & $(0,000)$ & 0,000 & $(0,000)$ \\
Zona central & $-0,006$ & $(0,683)$ & $-0,003$ & $(0,815)$ & 0,009 & $(0,166)$ \\
Zona este & 0,001 & $(0,913)$ & 0,055 & $(0,000)$ & $-0,057$ & $(0,000)$ \\
Zona noreste & 0,055 & $(0,000)$ & $-0,051$ & $(0,000)$ & $-0,004$ & $(0,248)$ \\
Zona noroeste & $-0,002$ & $(0,770)$ & 0,058 & $(0,000)$ & $-0,056$ & $(0,000)$ \\
Zona sur & 0,010 & $(0,278)$ & 0,023 & $(0,009)$ & $-0,032$ & $(0,000)$ \\
Residuos de la regresión & 0,146 & $(0,000)$ & $-0,152$ & $(0,000)$ & 0,005 & $(0,635)$ \\
auxiliar & & & & & & \\
Inverso del ratio de mills & 0,026 & $(0,063)$ & $-0,026$ & $(0,059)$ & 0,000 & $(0,972)$ \\
\hline
\end{tabular}

Nota: $*(\mathrm{p}<0,10), * *(\mathrm{p}<0,05), * * *(\mathrm{p}<0,01)$.

Fuente: Elaboración propia.

TABLA 7

ELASTICIDADES DEL MODELO QUAIDS CENSURADO

\begin{tabular}{|c|c|c|c|c|c|c|c|c|}
\hline & \multicolumn{2}{|c|}{ Elasticidad precio } & \multicolumn{2}{|c|}{$\begin{array}{l}\text { Elasticidad precio } \\
\text { compensada }\end{array}$} & \multicolumn{2}{|c|}{ Elasticidad gasto } & \multicolumn{2}{|c|}{ Elasticidad ingreso } \\
\hline & Promedio & $\begin{array}{l}\text { I. de C. } \\
\text { al } 95 \%\end{array}$ & Promedio & $\begin{array}{l}\text { I. de C. } \\
\text { al } 95 \%\end{array}$ & Promedio & $\begin{array}{l}\text { I .de C. } \\
\text { al } 95 \%\end{array}$ & Promedio & $\begin{array}{l}\text { I. de C. } \\
\text { al } 95 \%\end{array}$ \\
\hline Electricidad & $-0,846$ & $-0,931-0,761$ & $-0,580$ & $-0,626-0,534$ & 0,531 & $0,299 \quad 0,763$ & 0,101 & $0,047 \quad 0,154$ \\
\hline Gas natural & $-1,449$ & $-1,640-1,259$ & $-0,750$ & $-0,837-0,663$ & 1,842 & $1,559 \quad 2,126$ & 0,347 & $0,259 \quad 0,436$ \\
\hline Agua potable & $-0,759$ & $-0,794-0,723$ & $-0,724$ & $-0,781-0,666$ & 0,292 & $-0,185 \quad 0,768$ & 0,056 & $-0,038 \quad 0,151$ \\
\hline
\end{tabular}

Nota: Intervalo de Confianza (I. de C.). Fuente: Elaboración propia.

En general, la elasticidad precio de la demanda de electricidad estimada en este artículo se encuentra respaldada por los resultados de Blundell y Robin (1999), Gundimeda y Köhlin (2008) y Alberini et al. (2011) quienes analizan datos del Reino Unido, la India y en EE.UU., respectivamente. También existe 
similitud con los resultados de Labandeira et al. (2006) quienes analizan la demanda de energía en los hogares españoles.

Respecto del gas natural, nuestros resultados difieren con la literatura. La elasticidad precio del gas natural presente en la Tabla 7 supera, en valor absoluto, las correspondientes elasticidades de Blundell y Robin (1999) y Labandeira et al. (2006). Las razones de esta diferencia pueden ser los datos empleados, el diferente contexto económico o el tratamiento de la censura no incluido en los trabajos mencionados. En relación con el consumo de agua potable, la elasticidad precio obtenida en este trabajo es inelástica y, por tanto, está en armonía con lo señalado en la Tabla 1. Es sustancial destacar que Arbúes et al. (2004) y Martínez- Espiñeira $(2002,2007)$ estiman una demanda residencial de agua potable un tanto más inelástica que la señalada en la Tabla 7 . Sin embargo, el trabajo de Schleich y Hillenbrand (2009) presenta un resultado similar al nuestro.

Por otro lado, todas las elasticidades precio de las demandas compensadas tienen en valor absoluto, una magnitud menor a las elasticidades precio de la demanda. Esto implica que si los precios aumentan, los hogares serían más insensibles en el consumo de los bienes cuando la pérdida de poder adquisitivo se compensa mediante un incremento de la renta. De hecho, en el caso del gas natural, cuya demanda es la más sensible a las variaciones de su precio, la elasticidad precio compensada es prácticamente la mitad de su correspondiente elasticidad sin compensar.

Además, la Tabla 7 muestra que todos los bienes que forman los servicios básicos tienen una elasticidad gasto e ingreso de la demanda positiva. Debido a la magnitud de las elasticidades ingreso de la demanda se infiere que la electricidad, gas natural y agua potable serían considerados como bienes necesarios. Esto implica que un aumento del ingreso del hogar se traduce en un aumento del consumo de todos los bienes, pero en menor proporción. Sin embargo, los resultados muestran que no se rechazaría la hipótesis de una demanda de agua potable insensible a los cambios de su precio.

Las magnitudes de la elasticidad ingreso de la demanda de electricidad y agua potable se confirman observando los resultados de la Tabla 1. Los resultados de otros artículos como los de Filippini y Pachauri (2004), Hondroyiannis (2004) y Lee y Chiu (2011) confirman que la elasticidad ingreso de la demanda de electricidad es también positiva y menor a la unidad. Al igual que nuestros resultados, Arbúes et al. (2004) obtienen una elasticidad ingreso para la demanda de agua potable más cercana a cero. Sin embargo, para Ruijs et al. (2008) y Schleich y Hillenbrand (2009) esta demanda es más inelástica.

Respecto del gas natural, en este trabajo la elasticidad gasto es superior a lo que indican los resultados de Blundell y Robin (1999) y Labandeira et al. (2006). Por otro lado, si bien la elasticidad ingreso del gas natural es positiva y menor que la unidad, la Tabla 1 presenta una cifra para dicha sensibilidad mayor que Liao y Chang (2002) y Alberini et al. (2011). Tal como se ha comentado, estas diferencias pueden surgir debido a los datos utilizados, la crisis económica que afecta a los hogares españoles en 2012 y al tratamiento de la censura que surge del consumo del gas natural.

La Tabla 8 muestra cómo cambian las elasticidades precio para cada combustible residencial en diferentes tramos del ingreso residencial. Esta vez las cifras se obtienen para un hogar medio de la muestra en cada intervalo. Los resultados señalan que a medida que la renta aumenta, las elasticidades precio 
de la demanda de la electricidad, gas natural y agua potable se mantienen relativamente constantes en todas las familias de la muestra. Lo mismo ocurre con los intervalos de confianza.

Del mismo modo, la Tabla 9 muestra cómo varía la elasticidad ingreso de la demanda según la renta de los hogares de la muestra. Al igual que lo señalado en la Tabla 8 , los datos indican que las elasticidades ingreso se mantienen estables a medida que la renta familiar aumenta. Probablemente, la causa de este comportamiento de las elasticidades se debe a que todos los hogares medios en cada tramo de ingresos tienen una cuota de gasto similar en cada bien. En relación con los intervalos de confianza, no se aprecia una diferencia significativa entre los distintos tramos de ingreso mensual. Se destaca que no es posible rechazar, al $5 \%$ de significatividad, la hipótesis de una demanda de agua potable insensible a los cambios de su precio en todos los niveles de renta.

\section{Conclusiones}

El presente artículo examina el gasto de los hogares españoles en electricidad, gas natural y agua potable aplicando un modelo QUAIDS a los datos

\section{TABLA 8}

ELASTICIDADES PRECIO DE LA DEMANDA DE ELECTRICIDAD, GAS NATURAL Y AGUA POTABLE SEGÚN EL NIVEL DE INGRESO MENSUAL

\begin{tabular}{|c|c|c|c|c|c|c|}
\hline & \multicolumn{2}{|c|}{ Electricidad } & \multicolumn{2}{|c|}{ Gas natural } & \multicolumn{2}{|c|}{ Agua potable } \\
\hline & Promedio & $\begin{array}{l}\text { I. de C. } \\
\text { al } 95 \%\end{array}$ & Promedio & $\begin{array}{l}\text { I. de C. } \\
\text { al } 95 \%\end{array}$ & Promedio & $\begin{array}{l}\text { I. de C. } \\
\text { al } 95 \%\end{array}$ \\
\hline Menos de $1.000 € / \mathrm{mes}$ & $-0,862$ & $-0,963-0,760$ & $-1,457$ & $-1,658-1,257$ & $-0,775$ & $-0,808-0,742$ \\
\hline Entre 1.000 y $2.000 € / \mathrm{mes}$ & $-0,849$ & $-0,940-0,759$ & $-1,444$ & $-1,635-1,254$ & $-0,756$ & $-0,792-0,721$ \\
\hline Entre 1.000 y $3.000 € / \mathrm{mes}$ & $-0,844$ & $-0,925-0,763$ & $-1,450$ & $-1,639-1,261$ & $-0,750$ & $-0,786-0,713$ \\
\hline Más de $3.000 € /$ mes & $-0,835$ & $-0,911-0,759$ & $-1,451$ & $-1,637-1,266$ & $-0,763$ & $-0,797-0,728$ \\
\hline
\end{tabular}

Nota: Intervalo de Confianza (I. de C.). Fuente: Elaboración propia.

TABLA 9

ELASTICIDADES INGRESO DE LA DEMANDA DE ELECTRICIDAD, GAS NATURAL Y AGUA POTABLE SEGÚN EL NIVEL DE INGRESO MENSUAL

\begin{tabular}{|c|c|c|c|c|c|c|c|c|}
\hline & \multicolumn{3}{|c|}{ Electricidad } & \multicolumn{3}{|c|}{ Gas natural } & \multicolumn{2}{|c|}{ Agua potable } \\
\hline & Promedio & $\begin{array}{l}\text { I. de } \\
\text { al } 9\end{array}$ & C. & Promedio & $\begin{array}{l}\text { I. de } \\
\text { al } 9\end{array}$ & $\begin{array}{l}\mathrm{e} C . \\
5 \%\end{array}$ & Promedio & $\begin{array}{l}\text { I. de C. } \\
\text { al } 95 \%\end{array}$ \\
\hline Menos de $1.000 € / \mathrm{mes}$ & 0,105 & 0,047 & 0,163 & 0,349 & 0,254 & 0,444 & 0,058 & $-0,038 \quad 0,153$ \\
\hline Entre 1.000 y $2.000 € / \mathrm{mes}$ & 0,101 & 0,046 & 0,157 & 0,345 & 0,256 & 0,434 & 0,053 & $-0,045 \quad 0,150$ \\
\hline Entre 1.000 y $3.000 € / \mathrm{mes}$ & 0,100 & 0,048 & 0,153 & 0,348 & 0,261 & 0,435 & 0,053 & $-0,043 \quad 0,149$ \\
\hline Más de $3.000 € /$ mes & 0,105 & 0,047 & 0,163 & 0,349 & 0,254 & 0,444 & 0,058 & $-0,038 \quad 0,153$ \\
\hline
\end{tabular}

Nota: Intervalo de Confianza (I. de C.). Fuente: Elaboración propia. 
de la EPF de 2012. Este modelo es adaptado para incorporar la información sociodemográfica, el tratamiento de la posible endogeneidad del gasto total en los bienes del sistema y la censura en una variable explicada producto de la gran cantidad de hogares que no consumen gas natural. Entre las opciones disponibles para estimar un modelo de sistema de ecuaciones con censura, aplicamos el enfoque propuesto por Tauchmann (2010) que incorpora a cada ecuación del sistema un término de corrección generalizado basado en los trabajos de Heckman $(1976,1979)$. Debido a que solo una de las variables explicadas del modelo está censurada, dicho término de corrección se transforma en un inverso del ratio de Mills.

En relación con los resultados del trabajo, las elasticidades precio de la demanda para la electricidad y agua potable son, en valor absoluto, menores a la unidad. Esto significa que los hogares modifican la cantidad consumida de estos bienes en menor proporción a las variaciones de sus precios. Lo contrario sucede con el gas natural cuya demanda es elástica y, en consecuencia, un aumento de su precio disminuye la cantidad consumida en mayor proporción.

Al parecer, la mayor sensibilidad a los cambios en el precio del gas natural se genera porque los hogares podrían reemplazar dicho combustible por la electricidad. Probablemente, este combustible es considerado como la principal fuente de energía residencial: si el coste de ambos combustibles aumenta, es más fácil no utilizar la red de gas natural que la red eléctrica. Comparando los resultados de este artículo con otros que utilizan información de los hogares españoles, en particular con Arbúes et al. (2004), Martínez-Espiñeira (2002, 2007) y Labandeira et al. (2006), existe más similitud con las elasticidades precio de las demandas de electricidad y agua potable que con la demanda de gas natural. Esta diferencia puede tener su origen en los datos, la metodología econométrica aplicada o diferente contexto económico del país. Por lo demás, el examen de las elasticidades ingreso de la demanda de los bienes del sistema indica que la electricidad, gas natural y agua potable son considerados, en promedio, como bienes necesarios. No obstante, según lo intervalos de confianza, no es posible rechazar la presencia de una demanda de agua potable insensible a los cambios del ingreso familiar.

Los resultados de este trabajo pueden servir a quienes deben diseñar políticas que buscan promover un uso más eficiente de la energía y agua potable residencial. En tal sentido, si producto de la crisis económica y los elevados precios de la energía los hogares redujeran su consumo de electricidad y de gas natural, la disponibilidad de un aparato con mayor eficiencia energética, que reduce el coste del uso de dichos combustibles, ofrecería la oportunidad a las familias para recuperar sus antiguos niveles de consumo y satisfacción. De este modo, ya que la electricidad tiene una demanda inelástica, dicho incremento en el consumo de energía debería ser menor que en el caso de otros bienes que tienen una demanda elástica, tal como ocurre con el gas natural. En otras palabras, las elasticidades precio de la demanda proporcionan ciertos indicios sobre la efectividad del aumento de la eficiencia energética en la reducción del consumo de energía (Sorrell y Dimitropoulos, 2008). Además, si bien las elasticidades precio e ingreso se mantienen relativamente constantes en todos los hogares, es de esperar que aquellos con menores ingresos sean los más perjudicados cuando aumentan los precios de la energía y agua potable. Esto se puede deber a que el gasto en los bienes analizados, probablemente, repre- 
senta una mayor parte de la renta en los hogares con menor poder adquisitivo (Labandeira et al., 2006).

En el futuro, con el objeto de profundizar los resultados de este trabajo, sería recomendable examinar la evolución en el tiempo de las elasticidades precio e ingreso de la demanda de la electricidad, gas natural y agua potable. Con esta información se puede establecer una relación de mejor calidad entre el ambiente económico que afecta a los hogares españoles y el consumo residencial de los mencionados bienes.

\section{REFERENCIAS}

Alberini, Anna; Gans, Will y Velez-Lopez, Daniel (2011). "Residential Consumption of Gas and Electricity in the U.S.: The Role of Prices and Income", Energy Economics, Vol. 33; 870-881.

Amemiya, Takeshi (1974). "Multivariate Regression and Simultaneous Equation Models when the Dependent Variables are Truncated Normal", Econometrica, Vol. 42; 999-1012.

Arbúes, Fernando; Barberan, Ramón y Villanua, Inmaculada (2004). "Price Impact on Urban Residential Water Demand: A Dynamic Panel Data Approach", Water Resources Research, 40, W11402. doi: 10.1029/2004WR003092.

Arbúes, Fernando; Ángeles García-Valiñas, María y Martínez-Espiñeira, Roberto (2003). "Estimation of Residential Water Demand: A State-of-the-Art Review", The Journal of Socio-Economics, Vol. 32; 81-102.

Atuesta, L., y Paredes, D. (2012). "A Spatial Cost of Living Index for Colombia Using a Microeconomic Approach and Censored Data", Applied Economic Letters, Vol. 19; 1799-1805.

Banks, James; Blundell, Richard y Lewbel, Arthur (1997). "Quadratic Engel Curves and Consumer Demand", Review of Economics and Statistics, Vol. 79; 527-539.

Bilgic, Abdulbaki y Yen, Steven T. (2013). "Household Food Demand in Turkey: A Two-Step Demand System Approach", Food Policy, Vol. 43; 267-277.

Blundell, Richard y Robin, Jean Marc (1999). "Estimation in Large and Dissagregated Demand Systems: An Estimator for Conditionally Linear Systems", Journal of Applied Econometrics, Vol. 14; 209-232.

BOCG (2009). "Boletín Oficial de las Cortes Generales". Congreso de los Diputados, Serie D, Número 210 del 28/05/2009.

BOE (2012). “Real Decreto-Ley 13/2012”, Sec. I, Número 78 del 31/03/2012. Dagher, Leila (2012). "Natural Gas Demand at the Utility Level: An Application of Dynamic Elasticities", Energy Economics, Vol. 34; 961-969.

Deaton, Angus S y Muellbauer, John (1980). “An Almost Ideal Demand System”, American Economic Review, Vol. 70; 312-26.

Dong, Diansheng; Gould, Brian W. y Kaiser, Harry M. (2004). "Food Demand in Mexico: An application of the Amemiya-Tobin Approach to the Estimation of a Censored Food System", American Journal of Agricultural Economics, Vol. 86; 1097-1107.

Dubin, Jeffrey A. y McFadden, Daniel L. (1984). "An Econometric Analysis of Residential Electric Appliance Holdings and Consumption”, Econometrica, Vol. 52; 345-62. 
Eurostat (2014). "Statistic Database". Statistical Office of the European Communities. http://epp.eurostat.ec.europa.eu/. Último acceso: 20/05/2014. Filippini, Massimo y Pachauri, Shonali (2004). "Elasticities of Electricity Demand in Urban Indian Households", Energy Policy, Vol. 32; 429-436. Gundimeda, Haripriya y Köhlin, Gunnar (2008). "Fuel Demand Elasticities for Energy and Environmental Policies: Indian Sample Survey Evidence", Energy Economics, Vol. 30; 517-546.

Heckman, James J. (1976). "The Common Structure of Statistical Models of Truncation, Sample Selection and Limited Dependent Variables and a Simple Estimator for Such Models", Annals of Economic and Social Measurement, Vol. 5; 475- 492.

Heckman, James J. (1979). "Sample Selection Bias as a Specification Error", Econometrica, Vol. 47; 153-161.

Heien, Dale y Wessells, Cathy R. (1990). "Demand Systems Estimation with Microdata: A Censored Regression Approach", Journal of Business \& Economic Statistics, Vol. 8; 365-71.

Hoderlein, Stefan y Mihaleva, Sonya (2008). "Increasing the Price Variation in a Repeated Cross Section”, Journal of Econometrics, Vol. 147; 316-325. Hondroyiannis, George (2004). "Estimating Residential Demand for Electricity in Greece", Energy Economics, Vol. 26; 319-334.

Holt, Matthew T. y Goodwin, Barry K. (2009). "Contributions to Economic Analysis", Capítulo 2: The Almost Ideal and Translog Demand Systems, Emerald Group Publishing Limited, Vol. 288; 37-59.

Ibe, Oliver C. (2005). "Fundamentals of Applied Probability and Random Process", Elservier Academic Press, 1 Edición.

IDAE (2011). "Proyecto SECH-SPANHOUSEC: Análisis del Consumo Energético del Sector Residencial en España", Informe técnico, Instituto para la Diversificación y Ahorro de la Energía.

INE (2013). "Encuesta de Condiciones de Vida. Nota de Prensa", Instituto Nacional de Estadística. http://www.ine.es/prensa/np844.pdf.

INE (2014). "Índice de Precios de Consumo", Instituto Nacional de Estadística. http://www.ine.es/. Último acceso: 22/01/2014.

Kristrom, Bengt (2008). "Residential Energy Demand", OECD Journal: General Papers, Vol. 2008/2; 95-115.

Kuhn, H. W. y Tucker, A. W. (1951). "Nonlinear Programming". Proceedings of the Second Berkeley Symposium on Mathematical Statistics and Probability, University of California Press, Berkeley, Calif.; 481-492.

Labandeira, Xavier; Labeaga, José M. y Rodríguez, Miguel (2006). "A Residential Energy Demand System for Spain”, The Energy Journal, Vol. 27; 87-112. Lasarte, E., Rubiera, F. y Paredes, D. (2014). "City Size and Household Food Consumption: Demand Elasticities in Spain", Applied Economics, Vol. 46; 1624-1641.

Lee, Chien-Chiang y Chiu, Yi-Bin (2011). "Electricity Demand Elasticities and Temperature: Evidence from Panel Smooth Transition Regression with Instrumental Variable Approach", Energy Economics, Vol. 33; 896-902. Lee, Lung-Fei y Pitt, Mark M. (1986). "Microeconometric Demand Systems with Binding Nonnegativity Constraints: The Dual Approach", Econometrica, Vol. 54; 1237-1242. 
Liao, Huei-Chu y Chang, Tsai-Feng (2002). "Space-Heating and Water-Heating Energy Demands of the Aged in the U.S.", Energy Economics, Vol. 24; 267-284.

Madlener, Reinhard (1996). "Econometric Analysis of Residential Energy Demand: A Survey", Journal of Energy Literature II, Vol. 2; 3-32.

Martínez-Espiñeira, Roberto (2002). "Residential Water Demand in the NorthWest of Spain”, Enviromental and Resource Economics, Vol. 21; 161-187. Martínez-Espiñeira, Roberto (2007). "An Estimation of Residential Water Demand Using Co-Integration and Error Correction Techniques", Journal of Applied Economics, Vol. X; 161-184.

Nesbakken, Runa (2001). "Energy Consumption for Space Heating: A DiscreteContinuous Approach", Scandinavian Journal of Economics, Vol. 103; $165-84$.

Poi, B. P. (2008). “Demand-System Estimation: Update”. Stata Journal, Vol. 8; 554-556.

Pollak, Robert A. y Wales, Terence J. (1992). "Demand System Specification and Estimation", Oxford University Press, New York.

Ruijs, A.; Zimmermann, A. y Van den Berg, M. (2008). "Demand and Distributional Effects of Water Pricing Policies”, Ecological Economics, Vol. 66; 506-516.

Schleich, Joachim y Hillenbrand, Thomas (2009). "Determinants of Residential Water Demand in Germany”, Ecological Economics, Vol. 68; 1756-1769.

Shonkwiler, J. Scott y Yen, Steven T. (1999). "Two-Sstep Estimation of a Censored System of Equations", American Journal of Agricultural Economics, Vol. 81; 972-982.

Sorrell, Steve y Dimitropoulos, John (2008). "The Rebound Effect: Microeconomic Definitions, Limitations and Extensions”, Ecological Economics, Vol. 65; 636-649.

Tauchmann, Harald (2005). "Efficiency of Two-Step Estimators for Censored Systems of Equations: Shonkwiler and Yen Reconsidered", Applied Economics, Vol. 37; 367-374.

Tauchmann, Harald (2010). "Consistency of Heckman-Type Two-Step Estimators for the Multivariate Sample-Selection Model", Applied Economics, Vol. 42; 3895-3902.

Tobin, James (1958). "Estimation of Relationships for Limited Dependent Variables", Econometrica, Vol. 26; 24-36.

Vaage, Kjell (2000). "Heating Technology and Energy Use: A Discrete/Continuous Choice Approach to Norwegian Household Energy Demand", Energy Economics, Vol. 22; 649-666.

Wales, T. J. y Woodland, A. D. (1983). "Estimation of Consumer Demand Systems with Binding Non-Negativity Constraints", Journal of Econometrics, Vol. 21; 263-285.

Yen, Steven T.; Fang, Cheng y Su, Shew-Jiuan (2004). "Household Food Demand in Urban China: A Censored System Approach", Journal of Comparative Economics, Vol. 32; 564-585.

Zheng, Zhihao y Henneberry, Shida Rastegari (2010). "An Analysis of Food Grain Consumption in Urban Jiangsu Province of China", Journal of Agricultural and Applied Economics, Vol. 42; 337-355. 
Zheng, Zhihao y Henneberry, Shida Rastegari (2012). "Estimating the Impacts of Rising Food Prices on Nutrient Intake in Urban China”, China Economic Review, Vol. 23; 1090-1103. 